\title{
Intracellular evolution of mitochondrial DNA (mtDNA) and the tragedy of the cytoplasmic commons
}

\section{Citation}

Haig, David. 2016. "Intracellular Evolution of Mitochondrial DNA (mtDNA) and the Tragedy of the Cytoplasmic Commons." BioEssays 38 (6) (April 8): 549-555. doi:10.1002/bies.201600003.

\section{Published Version}

doi:10.1002/bies.201600003

\section{Permanent link}

http://nrs.harvard.edu/urn-3:HUL.InstRepos:34390138

\section{Terms of Use}

This article was downloaded from Harvard University's DASH repository, and is made available under the terms and conditions applicable to Open Access Policy Articles, as set forth at http:// nrs.harvard.edu/urn-3:HUL.InstRepos:dash.current.terms-of-use\#OAP

\section{Share Your Story}

The Harvard community has made this article openly available. Please share how this access benefits you. Submit a story.

Accessibility 


\title{
Do nuclear shepherds dream of mitochondrial sheep?
}

\section{Intracellular selection of mitochondrial DNA (mtDNA) and the tragedy of the cytoplasmic commons.}

\author{
David Haig \\ Department of Organismic and Evolutionary Biology, \\ Harvard University, 26 Oxford Street, \\ Cambridge MA 02138.
}

Keywords: aging, mtDNA, mitochondria, bottleneck, apoptosis, mitophagy, public goods 
Mitochondria exist in large numbers per cell. Therefore, the strength of natural selection on individual mtDNAs for their contribution to cellular fitness is weak whereas the strength of selection in favor of mtDNAs that increase their own replication without regard for cellular functions is strong. This problem has been solved for most mitochondrial genes by their transfer to the nucleus but a few critical genes remain encoded by mtDNA. Organisms manage the evolution of mtDNA to prevent mutational decay of essential services mitochondria provide to their hosts. Bottlenecks of mitochondrial numbers in female germlines increase the homogeneity of mtDNAs within cells and allow intraorganismal selection to eliminate cells with low quality mitochondria. Mechanisms of intracellular 'quality control' allow direct selection on the competence of individual mtDNAs. These processes maintain the integrity of mtDNAs within the germline but are inadequate to indefinitely maintain mitochondrial function in somatic cells. 


\section{Introduction}

The world population of human mitochondrial genomes (mtDNAs) is immense. Our planet is currently home to somewhat fewer than $10^{10}$ human bodies, each containing $10^{13}$ cells [1], each containing $10^{3}$ or more mtDNAs [2], for a rough estimate of $10^{26}$ total mtDNAs. Mitochondrial genetic variation is distributed within cells, among cells within bodies, and among bodies. Evolutionary processes of genetic drift and natural selection can occur at all these levels (Fig. 1) [3-5].

Mutation generates variation among mtDNAs within cells (heteroplasmy). A heteroplasmic herd of mtDNAs is subject to 'relaxed' replication, some mtDNAs are copied many times while others remain unreplicated, then mtDNAs are randomly sorted into daughter cells at mitosis. Such stochastic processes cause haplotype frequencies to drift within cell lineages, and to diverge among cells [6, 7]. But mtDNA is also subject to natural selection within and among cells. A variant that replicates faster than other members of its herd will increase its representation within a cell, and a cell lineage with a more efficient herd may generate daughter cells, with daughter herds, faster than cell lineages with less efficient herds [3]. Adaptive evolution of mtDNA for the benefit of bodies threatens to be overwhelmed by evolutionary processes within and among cells.

At first glance, mitochondrial efficiency should be subject to an insidious process of mutational degradation because purifying selection is ineffective at maintaining the function of large populations of interchangeable units that are judged by collective performance [8]. Suppose that a cell contains 1,000 copies of mtDNA and a mutation in one reduces that copy's contribution to oxidative phosphorylation by $10 \%$. The effect of this mutation is diluted a thousand-fold within the cell such that cellular efficiency is reduced by only $0.01 \%$, a shortfall that could be compensated by increasing mtDNA by a single copy until the next mutation that reduces cellular efficiency (and so on, in a ratchet-like process that steadily degrades mitochondrial function). By the same reasoning, positive selection for enhanced efficiency should be similarly ineffective because a mutation that increases efficiency by $10 \%$ would cause only a minuscule improvement in cellular function. In contrast, a mutant mtDNA that replicates $10 \%$ faster than nonmutant mtDNA in the same cell would increase steadily in frequency within its cell lineage even if mutant mtDNAs were associated with impaired cellular function. 
When viewed from this perspective, the maintenance of mitochondrial function is a classic problem of the provision of public goods. Contributions to cellular function benefit the cell, and all its inhabitants, but replication is a private good of individual mtDNAs. Thus, mtDNAs are subject to a tragedy of the cytoplasmic commons [9]. The risk of mutational decay of mtDNA is commonly ascribed to Muller's ratchet, the inexorable fixation of deleterious mutations in asexual lineages by random sampling from finite populations [3, 10, 11], but Muller's ratchet and the public-goods problem are distinct. Muller's ratchet turns more rapidly as population size decreases, but the public-goods problem is exacerbated as the number of mitochondria per cell increases. Social scientists have long recognized that public goods are easier to provide in smaller groups [12]. The mitochondrial public-goods problem appears to have been solved evolutionarily by a combination of mechanisms that reduce effective group size and convert oxidative phosphorylation into a private good of individual mtDNAs.

\section{Nuclear genes manage mitochondrial evolution for bodily benefit}

Mitochondria descend from an ancient endosymbiotic bacterium whose genome has dwindled by loss of non-essential genes and transfer of essential genes to the nucleus. About 1,000 genes are required to make a fully-functional mitochondrion, but mammalian mtDNA encodes only 13 membrane-bound proteins, two ribosomal RNAs, and 22 tRNAs. Most proteins essential for mitochondrial function are encoded by nuclear loci and shared among all of a cell's mitochondria $[13,14]$. Thus, the size of the group that provides each nuclear-encoded protein is reduced to a single gene in haploid cells, or two genes in diploid cells, with very

limited opportunities for within-cell selection. The performance of nuclear-encoded proteins is judged at the cellular or organismal level. Of particular importance, many of the genes responsible for replication of mtDNA, for fusion-fission cycles of mitochondria, and for apoptotic cell death, are nuclear-encoded and will have evolved to manage mtDNA populations for organismal benefit.

Nuclear genes are the herdsmen of domesticated mtDNAs. Our bodies possess multitudes of mitochondria in large herds within cells and myriad cellular herds within bodies. Because of these immense numbers, mitochondrial genomes are subject to significant evolution both within cells and among cells within bodies. If mitochondria reproduced at will within herds, without regard for cellular function, then the bioenergetic services mitochondria provide for their cellular and bodily hosts would steadily deteriorate, 
both within and across generations. Somatic herds, like the bodies they inhabit, deteriorate over time, but the next generation of bodies starts life with healthy herds. Nuclear genes have evolved to manage mitochondrial evolution for bodily benefit. Such management includes selective breeding of mitochondria with superior traits. Female germ cells are the stud farms that nurture and select the breeding stock that will populate the mitochondrial herds of the next generation.

\section{Bottlenecks increase mtDNA homogeneity within cells}

A body's mtDNAs are highly similar despite huge numbers in zygotes. This genetic homogeneity, together with large intergenerational shifts in the frequency of variant mtDNAs when these arise, provide evidence for one or more postzygotic restrictions of mitochondrial numbers [15]. Tight germline bottlenecks ensure that all copies of mtDNA in a matrilineal pedigree have a recent common ancestor and that heteroplasmy reflects recent mutation of mtDNA. Bottlenecks reduce the effective number of haplotypes segregating within cell lineages and lessen the public-goods problem because fewer distinct haplotypes contribute to (and share in) the cellular good of oxidative phosphorylation.

Bottlenecks redistribute variation in mtDNA from within to among cells, enhancing the effectiveness of natural selection on cellular function [16-18]. Although bottlenecks help maintain mtDNA quality for the long-term 'good of the species', this is an insufficient explanation for the evolution of bottlenecks. Nuclear and mitochondrial gene lineages part company every second generation, on average, when male-derived mtDNAs are eliminated from zygotes. Therefore, nuclear alleles that enforce a bottleneck, when maternally-derived, experience the same pool of mtDNAs, when paternally-derived, as alleles that fail to enforce a bottleneck. All share in the long-term benefit. For this reason, the selective maintenance of mitochondrial bottlenecks requires a short-term benefit for nuclear genes.

A bottleneck increases the average fitness of surviving offspring after selection but does not necessarily increase the average fitness of offspring before selection [16]. The solution to this conundrum is for selection to occur within female germlines before major maternal investment (Fig. 2). Parallel bottlenecks in multiple cell lineages of the germline increases mitochondrial variance among oocytes and provide a robust short-term advantage for nuclear genes if investment can be directed to oocytes of higher quality $[19,20]$. Such a redistributive process could occur before formation of primordial follicles, if 
primordial germ cells or oogonia with superior mtDNA have a proliferative advantage, or after formation of follicles, if oocytes of poor mitochondrial quality preferentially undergo atresia [21, 22]. Zygotic bottlenecks could serve the same purpose if mothers increase postzygotic care of superior offspring by selective abortion of low quality embryos.

In summary, germline bottlenecks reduce mitochondrial genetic variation within cells and increase variation among cells, thereby enhancing the effectiveness of cellular selection for mitochondrial function. Bottlenecks also decrease genetic variation of mtDNA within offspring bodies, thereby enhancing the effectiveness of organismal selection on mitochondrial traits. Cellular selection and organismal selection are likely to align for housekeeping functions of mtDNA, but soma-specific functions of mtDNA are not subject to selection within female germ lines. Germline selection is blind to strictly somatic functions, which will be selected only at the organismal level. Long-term evolution will therefore tend to favor soma-specific functions being performed by nuclear-encoded rather than mtDNA-encoded proteins. Indeed, mitochondrial proteins with tissue-specific isoforms are all nuclear-encoded [23].

\section{Quality control within cells eliminates defective mtDNA}

Cellular selection of mtDNA occurs when cells undergo differential proliferation determined by variation in mtDNA-encoded genes. The process becomes more efficient if slow growth is converted to no growth by elimination of cells with poor quality mtDNA. Selective cell death also allows selection of mtDNA to occur within populations of non-dividing cells. Apoptosis, among other functions, may eliminate cells with catastrophic mitochondrial failure to protect nearby cells from uncontrolled production of reactive oxygen species $[24,25]$.

Selection among cells is a blunt instrument to eliminate a few malfunctioning mitochondria. Sharper tools can be deployed to cull the lame but spare the herd. Mitochondria undergo cycles of fission and fusion within cells. Multiple mitochondria are distributed throughout the cytoplasm at mitosis. Mitochondria fuse to form large networks before entry to $\mathrm{S}$ phase and then the networks fragment before the next cell division $[26,27]$. Mitochondrial networks bud off parts, some of which are degraded, before surviving parts merge again with the network. This dynamic process of separation and reintegration is believed to function as a form of intracellular 'quality control' in which mitochondrial parts of lower membrane potential are 
eliminated by mitophagy [28-31]. Intracellular selection (mitophagy) and cellular selection (apoptosis) may work together to maintain the quality of mitochondrial herds.

Fusion can be conjectured to increase the effectiveness of quality control by equilibrating soluble factors among mitochondrial parts. By this means, fusion followed by fission establishes a 'level playing field' that isolates mtDNA as a factor responsible for variation in competence among mitochondrial parts. Such a selective mechanism requires that mtDNAs are physically linked to their gene products and included within the parts that undergo selective mitophagy [32, 33]. Of particular interest are 'kiss-and-run’ fusions in which soluble contents, but not nucleoids, are exchanged [34, 35]. tRNAs, unlike mitochondriallyencoded proteins and rRNAs, are not membrane-tethered, and are likely to be exchanged during fusion. Therefore, fission fragments of mitochondrial networks are likely to contain tRNAs encoded by multiple mtDNAs. For this reason, intracellular quality control should be less effective at eliminating deleterious mutations of tRNAs. Indeed, mutations in tRNA genes are disproportionately common causes of heritable mitochondrial disease [36].

Intracellular selection helps solve the public-goods problem by converting public goods into private goods. Each mtDNA must demonstrate its oxidative prowess to be accepted as a member of the group within its cell. In this way, 'compensation' of individual mtDNAs is tied to their 'performance'. Fission, fusion, and mitophagy undoubtedly have other functions to coordinate mitochondrial numbers with metabolic demand. But, if mtDNAs are to be eliminated for whatever reason, a selective process is better than a random process. Many questions remain about quality control, including whether mitochondria are eliminated because of failure to meet an absolute standard of performance or a comparative standard (how well a part performs relative to another part).

\section{mtDNAs evolve 'selfish' adaptations that promote their own replication}

The metabolic functions of mtDNA are adaptive for organisms and cells and are maintained, in part, by cellular and intracellular selection. But do mtDNAs also possess intracellular adaptations —independent of cellular function - that have evolved because of competition among mtDNAs within cells?

Intracellular selection favors mtDNAs that are superior competitors for a limited supply of replicases. Bottlenecks, in particular, create winners and losers. If passing through a bottleneck is likened to winning a 
lottery, then intracellular selection favors mtDNAs that purchase extra tickets. As a consequence, female germ cells may contain more copies of mtDNA than the number that would be justified by cellular function alone. Organismal-level selection of nuclear-encoded factors would then favor compensating reductions in mtDNA replication. However, nuclear genes face the evolutionary challenge of maintaining replicative order despite rampant polymorphism of mitochondrial haplotypes that results from clonal evolution in maternal lineages creating nested, independently-evolving, clades of mtDNA [37].

The control region, which contains origins of $\mathrm{H}$-strand replication, is the most rapidly evolving segment of mtDNA [38, 39]. Rapid evolution of the non-coding control region is commonly ascribed to relaxation of selective constraints relative to coding sequences [37] but positive selection to increase an mtDNA's representation within cells, and evade restraint by nuclear-encoded factors, should also be considered. Unfortunately, little is known about the regulation of mtDNA replication in female germlines.

Mitochondria move within cells by interactions with microtubule motors [40]. Some locations within germ cells may be more propitious than others for long term persistence of mtDNAs. If mtDNAs can influence their own movement then mitochondria might be expected to congregate at these propitious sites. For example, if the cytoplasmic distribution of replicases is anisotropic then mtDNAs would benefit from moving to, or failing to move from, sites with a relative abundance of replicases. Similarly, an oocyte's large stock of mitochondria is distributed among all cells of the early embryo, but only some cells become germ cell progenitors. Intracellular selection would favor mtDNA variants that had the knack of disproportionately segregating to germ cells rather than somatic cells.

Oocytes of animals with 'preformed' germlines, such as frogs, contain determinants of future germ cells ('germ plasm') intermingled with large numbers of mitochondria in a perinuclear agglomeration known as the mitochondrial cloud or Balbiani body [41]. A mtDNA that was able to segregate together with germ plasm would thereby increase its own representation in the germline. By comparison, oocytes of animals with 'epigenetic' determination of germ cells, such as mammals, may contain few spatial cues of the future location of germ cells [42]. Strategic self-placement by mtDNAs is possible only if mtDNAs can influence where and when they move, and such movement could be accomplished by microtubule motors fueled by locally-synthesized ATP. Movement of mitochondria into and out of the Balbiani body, based on the local delivery of fuel to mitochondrial motors, has been proposed as a mechanism of selecting 'healthy' 
mitochondria $[43,44]$. In this case, what is good for the mitochondrion is probably also adaptive at the cellular and organismal level.

Mitochondria are intimately involved in the initiation of apoptotic cell death [45, 46], a process that includes interactions between nuclear-encoded proteins, such as cytochrome $c$, and mtDNA-encoded proteins, such as subunits of cytochrome $c$ oxidase [47]. Because of segregation of mtDNAs among oocytes, a mtDNA that increases the chance that its oocyte avoids apoptosis potentially increases its representation in the next generation, but the same is not true for nuclear genes or for mtDNAs in surrounding follicle cells. Therefore, one might expect adaptations of mtDNA to avoid apoptosis in the female germline and compensating adaptations of nuclear-encoded genes, especially at molecular interfaces where their protein products interact.

\section{Mitochondrial breeding stock is actively managed in female germlines}

Nuclear genes are inherited equally via eggs and sperm but mtDNAs are transmitted predominantly or exclusively via eggs. Paternal transmission of mtDNA, if it occurs, must be exceedingly rare [48]. If one were to trace the forebears of a zygotic mtDNA backward in time, these ancestors would have resided in a recurrent sequence of zygotes, blastomeres, embryonic stem cells, primordial germ cells (PGCs), oogonia, and oocytes embedded exclusively (or overwhelmingly) within female bodies. For this reason, the long-term evolution of mtDNA sequences will have been jointly determined by evolutionary change within female germlines and by survival and reproduction of female bodies.

Fewer than a hundred extragonadal PGGs in an early embryo are the progenitors of all adult germ cells [49]. Descendants of these PGCs migrate to the developing ovary - roughly a thousand completing the trek — where oogonia proliferate before entering meiotic arrest as oocytes [50]. A subset of oocytes is surrounded by somatic cells to form primordial follicles and the remainder degenerates. From peak levels at mid pregnancy, follicles undergo progressive and continuous attrition until ovarian exhaustion at menopause. Of the 7 million follicles of a 20-week human fetus, 400,000 survive until birth, 200,000 remain at puberty, some hundreds are ovulated, and fewer than a thousand remain at menopause [51, 52]. During each ovarian cycle, a small cohort of follicles starts to grow, one follicle becomes dominant and the others cease growth and die. The oocyte of the dominant follicle finally completes meiosis - begun in the fetal 
ovary - after its fertilization in the fallopian tube. What sources of variation among oocytes could justify such profligacy? Variation among mitochondrial herds may be one contributing factor.

The zygotic endowment of mtDNAs does not resume replication until the blastocyst stage [53]. Therefore, the 200,000 or so copies of mtDNA in mature human oocytes [54] must be shared among many early embryonic cells. After this bottleneck, each pre-migratory human PGC contains fewer than ten mitochondria. The subsequent expansion of mitochondrial numbers is stupendous. Each oocyte of a primordial follicle contains about 6,000 copies of mtDNA. From a starting population of a few hundred mtDNAs in early PGCs, the 7 million human oocytes at mid-gestation are home to about 35 billion mitochondria! The final amplification of mtDNA within oocytes, that restores numbers to the 200,000 copies of the preceding maternal oocyte, occurs during the ovarian cycle before ovulation [19].

The demographies of female germ cells, and of their mitochondria, strongly suggest that the principal bottleneck of mtDNA numbers occurs before PGGs arrive at the germinal ridge, perhaps before differentiation of PGCs. Somatic cells can be experimentally induced to form pluripotent stem cells (iPSCs) that mimic cells of the early embryonic inner cell mass, and this process is accompanied by drastic reduction of mtDNA numbers that recapitulates an early embryonic bottleneck [55, 56]. It is unclear whether the mitochondrial bottlenecks of iPSCs reflect a passive process, diminution by cellular proliferation without mtDNA replication, or instead involve active degradation of mtDNAs.

Ample opportunities exist for cellular selection of mitochondrial function in the female germline. The gonadal migration of PGCs may be a strenuous test of ATP production for cells that set out with fewer than ten mitochondria: the proliferation of oogonia within ovaries may favor cell lineages with efficient mitochondrial herds; and metabolic testing of oocytes by follicles may weed out all but the best performing oocytes. (Follicle cells, it should be noted, are no more closely related to their own oocyte than to other oocytes of the female germline.) Finally, intracellular selection-quality control within oocytes — may also play a role [57].

Experimental data from mice, and the rarity of inherited mitochondrial disease relative to the agedependent accumulation of dysfunctional mtDNA in somatic cells, suggest that a selective sieve operates in female germlines to eliminate most, but not all, deleterious mtDNAs [57-59]. Despite purifying selection in the germline, there are persistent claims that mitochondrial function of oocytes deteriorates with age and 
contributes to the age-dependent decline of female fertility [60-62]. Although PGCs and oogonia are subject to cellular selection of migratory and proliferative competence, once fetal oocytes have entered meiosis, subsequent purifying selection is limited to quality control within cells and selective atresia of oocytes that, in older women, have not divided for decades. Mitochondrial aging of oocytes suggests that intracellular quality control and atretic losses from an exponentially-declining stock are insufficient to maintain mitochondrial function indefinitely in the face of disruptive forces of within-oocyte evolution.

\section{Mitochondrial function deteriorates with age in somatic cells}

The imperatives of mitochondrial husbandry differ for female germ cells and somatic cells. The overriding goal of germline husbandry is raising high-quality mitochondria for stocking the next generation of bodies.

By contrast, the overriding goal of somatic husbandry is the lifetime provision of mitochondrial services for the ultimate good of the germline. Somatic husbandry should include measures to maintain herd quality only to the extent that these measures advance the primary goal of germline support. Mitochondrial services may sometimes be provided more economically by 'cheap fixes' that deliver benefits now but compromise long-term somatic function.

Classes of mtDNA mutations present in aging somatic cells but absent in younger tissues suggest divergent evolutionary processes in germline and soma $[63,64]$. Some examples suggest ways in which adaptive management of mtDNA evolution might differ between germline and soma. First, germline bottlenecks facilitate selective breeding of functional mtDNAs, but similar bottlenecks in somatic tissues would come at the metabolic cost of temporarily reduced mitochondrial capacity. Second, oxidative phosphorylation in somatic cells can be maintained by functional complementation of mtDNAs that individually are unable to sustain function $[65,66]$, but complementation in the germline would lead to the evolutionary replacement of self-sufficient mtDNA lineages by heritable, heteroplasmic consortia that must segregate to daughter cells in correct proportions to maintain mitochondrial function. Third, increased replication of mtDNA in response to deficient electron transport (reactive biogenesis) is an adaptive response to immediate somatic needs that selectively favors less-efficient mtDNAs in the longer-term [24]. The short-term fitness benefits of reactive biogenesis may outweigh the long-term costs in somatic cells but would be problematic in the female germline. 
Somatic evolution of mtDNA inevitably proceeds by different paths in different tissues. Mitotic cell lineages are subject to cellular selection for faster division. Cellular selection might be a mechanism of maintaining functional herds of mitochondria for organismal benefit, but selection for cellular vigor is not without organismal risk, given the ever-present danger of cancer [67]. By contrast, evolution of mtDNA in postmitotic cell populations will be dominated by selection within cells and extinction of mitochondrial herds by unreplaced cell deaths. The $3243 \mathrm{~A}>\mathrm{G}$ mutation of a mitochondrial tRNA is the most common inherited cause of mitochondrial myopathy and is found at consistently higher levels in patients' skeletal muscle than in their peripheral blood. Moreover, mutation levels decrease with age in blood, but not muscle, suggesting purifying selection against mutant mtDNA in hematopoietic stem cells but not in postmitotic muscle $[68,69]$.

Mitochondrial function deteriorates with age, especially in post-mitotic tissues. This decline is associated with the accumulation of deleted and otherwise mutated mtDNAs [70]. Mitochondrial aging has been reported both in venerable mice and elderly humans despite large differences in body size and longevity [71]. Clearly, the rate of deterioration of mitochondrial function has been evolutionarily adjusted to species-specific lifespan. This suggests that mtDNA maintenance involves somatic trade-offs and that larger longer-lived animals invest more on maintenance of long-term mitochondrial performance [72]. Intracellular selection favors mtDNAs that replicate faster than other members of their herd. Variant mtDNAs that proliferate within herds despite impairment of cellular function are the mitochondrial analogues of cancers $[70,71]$.

\section{Conclusions and outlook}

The transfer of most mitochondrial genes to the nucleus circumvented the tragedy of the cytoplasmic commons for these genes, but organismal-level selection is relatively inefficient at maintaining cellular functions of the remaining mtDNA-encoded genes. This problem appears to have been solved by drastic reductions of mtDNA numbers within cells followed by reamplification. Such bottlenecks decrease mitochondrial variation within cells, but increase variation among cells, thereby enhancing the efficiency of cellular selection on mitochondrial function. Bottlenecks also increase mtDNA uniformity among the many cells of offspring, thus enhancing the efficiency of organismal selection. 
Intracellular selection of mtDNAs is seemingly more efficient than cellular or organismal selection because individual mtDNAs are judged directly by their metabolic performance. Why bother with bottlenecks if the same end can be achieved by intracellular quality control? Poultry breeding suggests an answer. Chicken, like mitochondria, are raised in groups. Artificial selection that chooses parents based on individual performance has perpetuated behaviors that increase individual performance at the expense of the performance of other group members. Chicken breeders have been able to increase collective yield by selection on group performance (analogous to cellular selection) rather than individual performance [75].

The contributions of mtDNA to cellular fitness within female germlines and to organismal fitness are likely to align for housekeeping functions, but soma-specific adaptations of mtDNA are not subject to intracellular or cellular selection within female germlines. Over the long-term course of evolution one might expect mtDNA to become specialized in housekeeping roles, and somatic adaptations to be delegated to nuclear control. Mitochondria have been thoroughly domesticated, and their reproduction tightly controlled by nuclear genes, but selfish evolution of mtDNA within germline herds has not been altogether eliminated. Mitonuclear conflicts within the female germline are likely to be expressed over the control of mtDNA replication, mitophagy, and apoptosis.

Acknowledgments: The manuscript has benefited from the comments of Christopher W. Baker, Yaniv Brandvain, Andrew Moore, Pavitra Muralidhar, Stephen Stearns, and the anonymous reviewers. 
1. Bianconi E, Piovesan A, Facchin F, Beraudi A, et al. 2013. An estimation of the number of cells in the human body. Ann Hum Biol 40: 463-71.

2. Reis RJS, Goldstein S. 1983. Mitochondrial DNA in mortal and immortal human cells. J Biol Chem 258: $9076-85$.

3. Rand DM. 2001. The units of selection of mitochondrial DNA. Annu Rev Ecol Syst 32: 415-48.

4. Taylor DR, Zeyl C, Gooke E. 2002. Conflicting levels of selection in the accumulation of mitochondrial defects in Saccharomyces cerevisiae. Proc Natl Acad Sci USA 99: 3690-4.

5. Aanen DK, Spelbrink JN, Beekman M. 2014. What cost mitochondria? The maintenance of functional mitochondrial DNA within and across generations. Phil Trans R Soc B 369: 20130438.

6. Ghinnery PF, Samuels DC. 1999. Relaxed replication of mtDNA: a model with implications for the expression of disease. Am $\mathcal{F}$ Hum Genet 64: 1158-65.

7. Johnston IG, Burgstaller JP, Havlicek V, Kolbe T, et al. 2015. Stochastic modelling, Bayesian inference, and new in vivo measurements elucidate the debated mtDNA bottleneck mechanism. eLife 4: e07464.

8. Kondrashov AS. 1994. Mutation load under vegetative reproduction and cytoplasmic inheritance. Genetics 137: 311-8.

9. Hardin G. 1968. The tragedy of the commons. Science 162: 1243-8.

10. Lynch M. 1996. Mutation accumulation in transfer RNAs: molecular evidence for Muller's ratchet in mitochondrial genomes. Mol Biol Evol 13: 209-220.

11. Havird JC, Hall MD, Dowling DK. 2015. The evolution of sex: a new hypothesis based on mitochondrial mutational erosion. Bioessays 37: 951-8.

12. Olson M. 1961. The logic of collective action. Public goods and the theory of groups. Harvard University Press.

13. Goffart S, Wiesner RJ. 2003. Regulation and co-ordination of nuclear gene expression during mitochondrial biogenesis. Exp Physiol 88: 33-40.

14. Falkenberg M, Larsson NG, Gustafsson GM. 2007. DNA replication and transcription in mammalian mitochondria. Annu Rev Biochem 76: 679-99.

15. Wallace DG, Ghalkia D. 2013. Mitochondrial DNA genetics and the heteroplasmy conundrum in evolution and disease. Cold Spring Harbor Persp Biol 5: a021220. 
16. Bergstrom GT, Pritchard J. 1998. Germline bottlenecks and the evolutionary maintenance of mitochondrial genomes. Genetics 149: 2135-46.

17. Roze D, Rousset F, Michalakis Y. 2005. Germline bottlenecks, biparental inheritance and selection on mitochondrial variants: a two-level selection model. Genetics 170: 1385-99.

18. Hadjivasiliou Z, Pomiankowski A, Seymour RM, Lane N. 2012. Selection for mitonuclear coadaptation could favour the evolution of two sexes. Proc $R$ Soc B 279: 1865-72.

19. Jansen RPS, de Boer K. 1998. The bottleneck: mitochondrial imperatives in oogenesis and ovarian follicular fate. Mol Cell Endocrinol 145: 81-8.

20. Stearns SC, Ebert D. 2001. Evolution in health and disease: work in progress. QRev Biol 76: 417-32.

21. Krakauer DG, Mira A. 1999. Mitochondria and germ cell death. Nature 400: 125-6.

22. Krakauer DG, Mira A. 2000. Mitochondria and the death of oocytes. Nature 403: 501.

23. Pierron D, Wildman DE, Hüttemann M, Markondapatnaikuni GC, et al. 2012. Gytochrome c oxidase: evolution of control via nuclear subunit addition. Biochim Biophys Acta 1817: 590-7.

24. Lane N. 2011. Mitonuclear match: optimizing fitness and fertility over generations drives ageing within generations. Bioessays 33: 860-9.

25. Dibrova DV, Gherepanov DA, Galperin MY, Skulachev VP, Mulkidjanian AY. 2013.

Evolution of cytochrome $b c$ complexes: from membrane-anchored dehydrogenases of ancient bacteria to triggers of apoptosis in vertebrates. Biochim Biophys Acta 1827: 1407-27.

26. Mitra K, Wunder G, Roysam B, Lin G, Lippincott-Schwartz J. 2009. A hyperfused mitochondrial state achieved at $\mathrm{G}_{1}-\mathrm{S}$ regulates cyclin $\mathrm{E}$ buildup and entry into $\mathrm{S}$ phase. Proc Natl Acad Sci USA 106: 11960-5.

27. Mitra K. 2013. Mitochondrial fission-fusion as an emerging key regulator of cell proliferation and differentiation. Bioessays 35: 955-64.

28. Twig G, Hyde B, Shirihai OS. 2008. Mitochondrial fusion, fission and autophagy as a quality control axis: the bioenergetic view. Biochim Biophys Acta 1777: 1092-7.

29. Suen DF, Narendra DP, Tanaka A, Manfredi G, Youle RJ. 2010. Parkin overexpression selects against a deleterious mtDNA mutation in heteroplasmic cybrid cells. Proc Natl Acad Sci USA 107: 1183540. 
30. Osman G, Noriega TR, Okreglak V, FungJG, Walter P. 2015. Integrity of the yeast mitochondrial genome, but not its distribution and inheritance, relies on mitochondrial fission and fusion. Proc Natl Acad Sci USA 112: 11835-40.

31. Hoitzing H, Johnston IG, Jones NS. 2015. What is the function of mitochondrial networks? A theoretical assessment of hypotheses and proposal for future research. Bioessays 37: 687-700.

32. Kowald A, Kirkwood TBL. 2011. Evolution of the mitochondrial fusion-fission cycle and its role in aging. Proc Natl Acad Sci USA 108: 10237-42.

33. Busch KB, Kowald A, Spelbrink JN. 2014. Quality matters: how does mitochondrial network dynamics and quality control impact on mtDNA integrity? Phil Trans R Soc B 369: 20130442.

34. Liu X, Weaver D, Shirihai O, Hajnóczky G. 2009. Mitochondrial 'kiss-and-run': interplay between mitochondrial motility and fusion-fission dynamics. EMBO 7 28: 3074-89.

35. Yang L, Long Q, Liu J, Tang H, et al. 2015. Mitochondrial fusion provides an 'initial metabolic complementation' controlled by mtDNA. Cell Mol Life Sci 72: 2585-98.

36. Holt IJ, Speijer D, Kirkwood TBL. 2014. The road to rack and ruin: selecting deleterious mitochondrial DNA variants. Phil Trans $R$ Soc B 369: 20130451.

37. van Oven M, Kayser M. 2008. Updated comprehensive phylogenetic tree of global human mitochondrial DNA variation. Hum Mutation 30: E386-94.

38. Horai S, Hayasaka K. 1990. Intraspecific nucleotide sequence differences in the major noncoding region of human mitochondrial DNA. Am 7 Hum Genet 46: 828-42.

39. Stoneking M, Hedgecock D, Higuchi RG, Vigilant L, Erlich HA. 1991. Population variation of human mtDNA control region sequences detected by enzymatic amplification and sequence-specific oligonucleotide probes. Am 7 Hum Genet 48: 370-82.

40. Boldogh IR, Pon LA. 2007. Mitochondria on the move. Trends Cell Biol 17: 502-10.

41. Kloc M, Bilinski SM, Etkin LD. 2004. The Balbiani body and germ cell determinants: 150 years later. Curr Top Devel Biol 59: 1-36.

42. Extavour GG, Akam M. 2003. Mechanisms of germ cell specification across the metazoans: epigenesis and preformation. Development 130: 5869-84. 
43. Zhou RR, Wang B, WangJ, Schatten H, Zhang YZ. 2010. Is the mitochondrial cloud the selection machinery for preferentially transmitting wild-type mtDNA between generations? Rewinding Müller's ratchet efficiently. Curr Genet 56: 101-7.

44. Milani L. 2015. Mitochondrial membrane potential: a trait involved in organelle inheritance? Biol Lett 11: 20150732.

45. Desagher S, Martinou JC. 2000. Mitochondria as the central control point of apoptosis. Trends Cell Biol 10: 369-77.

46. Martinou JC, Youle RJ. 2011. Mitochondria in apoptosis: Bcl-2 family members and mitochondrial dynamics. Devel Cell 21: 92-101.

47. Hütteman M, Helling S, Sanderson TH, Sinkler G, et al. 2012. Regulation of mitochondrial respiration and apoptosis through cell signaling: cytochrome $c$ oxidase and cytochrome $c$ in ischemia/reperfusion injury and inflammation. Biochim Biophys Acta 1817: 598-609.

48. Pyle A, Hudson G, Wilson IJ, Coxhead J, et al. 2015. Extreme-depth re-sequencing of mitochondrial DNA finds no evidence of paternal transmission in humans. PLoS Genet 11: e1005040.

49. Lawson KA, Hage WJ. 1994. Clonal analysis of the origin of primordial germ cells in the mouse. In Marsh J, Goode J. ed; Germline development. John Wiley. p. 68-91.

50. Gosden RG. 1985. Biology of menopause. Academic Press.

51. McGee EA, Hsueh AJW. 2000. Initial and cyclic recruitment of ovarian follicles. Endocrine Rev 21: $200-14$

52. Broekmans FJ, Soules MR, Fauser BC. 2009. Ovarian aging: mechanisms and clinical consequences. Endocrine Rev 30: 465-93.

53. Thundathil J, Filion F, Smith LC. 2005. Molecular control of mitochondrial function in preimplantation mouse embryos. Mol Reprod Devel 71: 405-13.

54. Reynier P, May-Panloup P, Ghrétien MF, Morgan CY, et al. 2007. Mitochondrial DNA content affects the fertilizability of human oocytes. Mol Hum Reprod 7: 425-9.

55. Prigione A, Fauler B, Lurz R, Lehrach H, Adjaye J. 2010. The senescence-related mitochondrial/oxidative stress pathway is repressed in human induced pluripotent stem cells. Stem Cells 28: $721-33$. 
56. Folmes CDL, Martinez-Fernandez A, Perales-Clemente E, Li X, et al. 2013. Disease-causing mitochondrial heteroplasmy segregated within induced pluripotent stem cell clones derived from a patient with MELAS. Stem Cells 31: 1298-308.

57. Stewart JB, Freyer G, Elson JL, Larsson NG. 2008. Purifying selection of mtDNA and its implications for understanding evolution and mitochondrial disease. Nature Rev Genet 9: 657-62.

58. Stewart JB, Freyer G, Elson JL, Wredenberg A, et al. 2008. Strong purifying selection in transmission of mammalian mitochondrial DNA. PLoS Biol 6: e10.

59. Fan W, Waymire KG, Narula N, Li P, et al. 2008. A mouse model of mitochondrial disease reveals germline selection against severe mtDNA mutations. Science 319: 958-62.

60. Tarín JJ. 1996. Potential effects of age-associated oxidative stress on mammalian oocytes/embryos. Mol Hum Reprod 2: 717-24.

61. Wilding M, Dale B, Marino M, di Matteo L, et al. 2001. Mitochondrial aggregation patterns and activity in human oocytes and preimplantation embryos. Hum Reprod 16: 909-17.

62. Eichenlaub-Ritter U, Wieczorek M, Lüke S, Seidel T. 2011. Age related changes in mitochondrial function and new approaches to study redox regulation in mammalian oocytes in response to age or maturation conditions. Mitochondrion 11: 783-96.

63. Greaves LG, Elson JL, Nooteboom M, Grady JP, et al. 2012. Comparison of mitochondrial mutation spectra in ageing human colonic epithelium and disease: absence of evidence for purifying selection in somatic mitochondrial DNA point mutations. PLoS Genet 8: e1003082.

64. Kowald A, Kirkwood TBL. 2014. Transcription could be the key to the selection advantage of mitochondrial deletion mutants in aging. Proc Natl Acad Sci USA 111: 2972-7.

65. Inoue K, Nakada K, Ono T, Isobe K, et al. 2001. Inter-mitochondrial complementation: mitochondria-specific system preventing mice from expression of disease phenotypes by mutant mtDNA. Nature Med 7: 934-40.

66. Gilkerson RK, Schon EA, Hernandez E, Davidson MM. 2008. Mitochondrial nucleoids maintain genetic autonomy but allow for functional complementation. 7 Cell Biol 181: 1117-28.

67. Zhidkov I, Livneh EA, Rubin E, Mishmar D. 2009. mtDNA mutation pattern in tumors and human evolution are shaped by similar selective constraints. Genome Res 19: 576-80. 
68. Frederiksen AL, Andersen PH, Kyvik KO, Jeppesen TD, et al. 2006. Tissue-specific distribution of the $3243 \mathrm{~A} \rightarrow \mathrm{G}$ mtDNA mutation. 7 Med Genet 43: 671-7.

69. Rajasimha HK, Chinnery PF, Samuels DG. 2008. Selection against pathogenic mtDNA mutations in a stem cell population leads to the loss of the $3243 \mathrm{~A} \rightarrow \mathrm{G}$ mutation in blood. Am 7 Hum Genet 82: 333-43.

70. Payne BAI, Ghinnery PF. 2015. Mitochondrial dysfunction in aging: much progress but many unresolved questions. Biochim Biophys Acta 1847: 1347-53.

71. Larsson NG. 2010. Somatic mitochondrial DNA mutations in mammalian aging. Annu Rev Biochem 79: $683-706$.

72. Kowald A, Kirkwood TBL. 2013. Mitochondrial mutations and aging: random drift is insufficient to explain the accumulation of mitochondrial deletion mutants in short-lived animals. Aging Cell 12: 72831.

73. Moraes CT, Kenyon L, Hao H. 1999. Mechanisms of human mitochondrial DNA maintenance: the determining role of primary sequence and length over function. Mol Biol Cell 10: 3345-56.

74. Diaz F, Bayona-Bafaluy MP, Rana M, Mora M, et al. 2002. Human mitochondrial DNA with large deletions repopulates organelles faster than full-length genomes under relaxed copy number control. Nucleic Acids Res 30: 4626-33.

75. Muir WM. 1996. Group selection for adaptation to multi-hen cages: selection program and direct responses. Poultry Sci 75: 447-58. 
Figure 1: Mitochondria (colored circles) are contained within cells (inner envelopes) and cells are contained within bodies (outer envelopes). Mitochondrial differences exist within cells, among cells within bodies, and among bodies. Natural selection and genetic drift can act at all these levels. Mutation generates new variants within cells.

Figure 2: A cell (a) is heteroplasmic for blue and red mitochondria. The cell divides to produce descendant cells without a bottleneck (b) or with a bottleneck (b*). Genetic drift occurs at each cell division. The effect of the bottleneck is to accentuate drift and increase the differences among cells (compare c and $\mathrm{c}^{*}$ ). The effect of cellular selection is represented at $\mathrm{d}$ and $\mathrm{d}^{*}$ (the cell with the most blue mitochondria was eliminated and replaced by a descendant of the cell with the fewest blue mitochondria). 